\title{
On the voltage dependence of sensitivity for Schottky-type gas sensor
}

\author{
Y. Liu, ${ }^{1}$ J. Yu, ${ }^{1}$ W. M. Tang ${ }^{2}$ and P. T. Lai ${ }^{1, a)}$ \\ ${ }^{1}$ Department of Electrical and Electronic Engineering, The University of Hong Kong, Pokfulam Road, \\ Hong Kong \\ ${ }^{2}$ Department of Applied Physics, The Hong Kong Polytechnic University, Hung Hom, Kowloon, Hong Kong
}

(Received 9 September 2014; accepted 18 November 2014; published online 2 December 2014)

\begin{abstract}
The voltage dependence of sensitivity for Schottky-diode gas sensor is theoretically studied and experimentally verified in this work. The study is based on the forward current-voltage $(I-V)$ characteristics of the device and benefits from its power exponent parameter $\alpha(V)=[\mathrm{d}(\ln I)] /[\mathrm{d}(\ln V)]$. This proposed analytical method (1) provides an easier way to identify the current-flow mechanisms at different bias levels, (2) demonstrates the exponential relation between the device sensitivity and the voltage at low bias level, (3) allows easy and accurate calculation of the Schottky barrier-height change, and (4) lays the groundwork for investigating the maximum sensitivity and the corresponding bias voltage. This analytical method is verified by using a $\mathrm{Pd} / \mathrm{WO}_{3} / \mathrm{SiC}$ diode under exposure to hydrogen gas with different concentrations at $150^{\circ} \mathrm{C}$ and $225^{\circ} \mathrm{C}$. Based on the proposed method, the parameters (barrier-height change, maximum sensitivity and corresponding bias voltage) of the sensor can be easily extracted and show excellent consistence with those obtained by conventional method. (C) 2014 AIP Publishing LLC.
\end{abstract}

[http://dx.doi.org/10.1063/1.4903231]

Since the Schottky-diode hydrogen sensor was first reported by Shivaraman and Lundström et al. in 1976, ${ }^{1}$ there has been tremendous development on metal-semiconductortype gas sensors due to their simplicity, portability, and high sensitivity. $^{2-5}$ Typically, Schottky-barrier-based devices are fabricated by combining catalytic metal, such as palladium or platinum, with semiconductor substrate (MS). Some also consist of a very thin insulating or oxide layer, generally several nanometers, between the metal and semiconductor layer (MIS or MOS). The principal mechanism for such gas detectors has been well described in terms of a change in Schottky barrier height when exposed to a target gas. In this work, the classical case of hydrogen sensors will be adopted as an example.

As hydrogen molecules adsorb on a transition-metal surface, they first dissociate to hydrogen atoms via catalytic reaction. Some of the atoms diffuse through the metal film and form a dipole layer at the metal-insulator interface due to polarization by internal electric field. Such a dipole layer causes an extra voltage drop and as a result reduces the Schottky barrier height. Therefore, the hydrogen concentration in the ambience can be determined by an increase of current in the current-voltage $(I-V)$ characteristics. ${ }^{3}$

Using the classical thermal emission theory, some important physical parameters of Schottky diodes can be extracted, such as ideality factor $(n)$, series resistance $\left(R_{\mathrm{s}}\right)$, and zero-biased barrier height $\left(\phi_{\mathrm{B} 0}\right)$ if provided with a known Richardson constant. Subsequently, changes in the parameters can be quantitatively compared before and after the device is introduced to hydrogen gas. Another commonly used reference to evaluate the performance is sensitivity $(S)$, defined as the relative change of current at a given voltage bias. The data analysis techniques in most gas sensor publications, however, have several common noticeable

\footnotetext{
a) Author to whom correspondence should be addressed. Electronic mail: laip@eee.hku.hk.
}

limitations: (1) Over-simplification of the thermal emission theory and ignoring the contribution of parallel (or shunt) resistance due to electron accumulation and localized states at the oxide-semiconductor interface. ${ }^{6}$ Most of the studied devices today are fabricated on $\mathrm{SiC}$ or GaN substrate, and the barrier height is larger than $0.83 \mathrm{eV}{ }^{7}$ Therefore, the parallel resistance needs to be considered-especially at low voltage bias and be careful with the change of dominant current flow mechanism after exposing to target gases; (2) The assumption that the diode parameters are constant or linear over the studied bias region. However, for real diodes, the ideality factor, barrier height and series or parallel resistance all show bias dependence; ${ }^{8}$ (3) Few publications have studied the relation between gas sensitivity and voltage bias or explained how to choose a suitable bias voltage for the best sensing performance; (4) two or more stages of graphical parameter extraction from the $I-V$ characteristics are required to obtain the barrier-height change.

To simplify the methodology as mentioned above, this work proposes and validates the exponential relation between sensitivity and voltage $(S-V)$ based on the Schottky diode equation. By introducing the power exponent parameter,

$$
\alpha=\frac{d(\ln I)}{d(\ln V)},
$$

the bias voltage for the device to operate under maximum sensitivity condition can be determined. Besides, the barrierheight change can be easily obtained without two stages of parameter extraction. This transformation technique was first demonstrated by G. Bagratishvili et al., ${ }^{9}$ and it permits to trace the change in current-transport mechanism at different bias levels. In this work, the effect of parallel resistance on the Schottky diode in nitrogen gas is examined and turns out to be insignificant after the diode is inserted in hydrogen gas due to the reduced barrier height. Once the current flow mechanism is confirmed and the diode parameters $n, I_{\mathrm{S}}, R_{\mathrm{S}}$ in 
hydrogen are extracted, the maximum sensitivity and the corresponding applied voltage can be predicted. All the analysis is carried out with the experimental $I-V$ data of a gas sensor based on $\mathrm{Pd} / \mathrm{WO}_{3} / \mathrm{SiC}$ structure.

Based on the classical thermal emission model, the current dependence on applied voltage of a Schottky diode can be expressed as follows: ${ }^{10}$

$$
I=I_{0}\left[\exp \left(\frac{q\left(V-I R_{s}\right)}{n k T}\right)-1\right],
$$

where $I_{0}$ is the saturation current and is given by

$$
I_{0}=A A^{*} T^{2}\left[\exp \left(-\frac{q \phi_{B 0}}{k T}\right)\right] .
$$

$V, q, k$, and $T$ are the applied voltage, electron charge, Boltzmann constant and absolute temperature, respectively. $A$ is the Schottky-contact area and $A^{*}$ is the effective Richardson constant. $R_{\mathrm{S}}$ is the series resistance of the neutral region of the semiconductor bulk. $\phi_{\mathrm{B} 0}$ is the zero-biased barrier height and $n$ is the ideality factor.

Using Eq. (2), the power exponent parameter $(\alpha)$ defined by Eq. (1) becomes

$$
\alpha=\frac{q V}{n k T+q I R_{s}} .
$$

For Schottky-diode gas sensor, the sensitivity is defined as

$$
S=\frac{I_{H 2}-I_{N 2}}{I_{N 2}} \approx \frac{I_{H 2}}{I_{N 2}}, \text { for } \quad S \gg 1 .
$$

Taking a simple differentiation of logarithm of sensitivity with respect to the voltage,

$$
\frac{d \ln S}{d V}=\frac{1}{V}\left(\alpha_{H}-\alpha_{N}\right) .
$$

The subscripts $H$ and $N$ denote the parameters in hydrogen and nitrogen ambience, respectively.

Under low-bias condition where $V \gg I R_{\mathrm{s}}$, Eq. (4) can be written as

$$
\alpha=\frac{q V}{n k T}
$$

and Eq. (6) can be rewritten as

$$
\frac{d \ln S}{d V}=\frac{q}{k T}\left(\frac{1}{n_{H}}-\frac{1}{n_{N}}\right) .
$$

By integration,

$$
\ln S=-\frac{q}{k T} \Delta \phi_{B 0}+\frac{q}{k T}\left(\frac{1}{n_{H}}-\frac{1}{n_{N}}\right) V .
$$

Therefore, the sensitivity has a simple exponential relation with voltage in the low-bias regime, if the ideality factor is assumed to be independent of the voltage. Moreover, the barrier-height change $\Delta \phi_{\mathrm{B} 0}$ is revealed immediately from the $y$-intercept of a $\ln S-V$ plot without extra calculations, and the variation in ideality factor can be determined from the slope of the plot.

Experimental verification is applied on a $\mathrm{Pd} / \mathrm{WO}_{3} / \mathrm{SiC}$ MOS-type Schottky-diode gas sensor. The whole structure consists of a 100-nm palladium Schottky pad (contact area $\mathrm{A}=0.3 \mathrm{~mm}^{2}$ ) and a $10-\mathrm{nm} \mathrm{WO}_{3}$ film sputtered on an $n$-type $4 \mathrm{H}-\mathrm{SiC}$ substrate. The backside is covered with $20-\mathrm{nm} \mathrm{Ti} /$ 80-nm Au layers by e-beam evaporation as ohmic contact. Details of the fabrication procedure are described in previous work. $^{11}$

Figs. 1(a) and 1(b) show the experimental $I-V$ characteristics measured under exposure to pure nitrogen and diluted hydrogen gas in nitrogen with four concentrations: $40 \mathrm{ppm}$, $350 \mathrm{ppm}, 675 \mathrm{ppm}$, and $1000 \mathrm{ppm} \mathrm{H}_{2} / \mathrm{N}_{2}$ at $150{ }^{\circ} \mathrm{C}$ and $225^{\circ} \mathrm{C}$, respectively. The inset figures are corresponding $I-V$ data in linear scale, and the SiC-based gas sensor exhibits a good rectifying behavior in both $\mathrm{N}_{2}$ and $\mathrm{H}_{2} / \mathrm{N}_{2}$ gases. From the slope of the $I-V$ curve in the forward-bias region, the current-flow mechanism can be divided into three parts, parallel-resistance-controlled linear region (very low current), thermal-emission exponential region (moderate current), and series-resistance-controlled region (high current). ${ }^{12}$

By fitting the measured $I-V$ data in the exponential part with Eqs. (2) and (3), the zero-biased barrier height $\phi_{\mathrm{B} 0}$ and ideality factor $(n)$ are extracted and listed in Table I. After exposing to hydrogen, the barrier height is reduced and the total flow current grows. The maximum barrier-height change corresponding to $1000-\mathrm{ppm} \mathrm{H}_{2} / \mathrm{N}_{2}$ at $150{ }^{\circ} \mathrm{C}$ and $225^{\circ} \mathrm{C}$ is $160 \mathrm{meV}$ and $220 \mathrm{meV}$, respectively.

With the definition in Eq. (5), the sensitivity and voltage $(S-V)$ characteristics for 40-ppm and 1000-ppm $\mathrm{H}_{2} / \mathrm{N}_{2}$ at $150{ }^{\circ} \mathrm{C}$ and $225^{\circ} \mathrm{C}$ are drawn in Fig. 2. The sensitivity first increases with applied voltage and reaches the highest point at $0.75 \mathrm{~V}$ and then decreases slowly. This trend is observed for all the concentrations and both temperatures. The rising section $(0.34 \mathrm{~V}-0.52 \mathrm{~V})$ of the $S-V$ curve in semi-logarithm scale is analyzed by linear fitting, as indicated with solid line in Fig. 2. The R-square value is found to be larger than 0.99
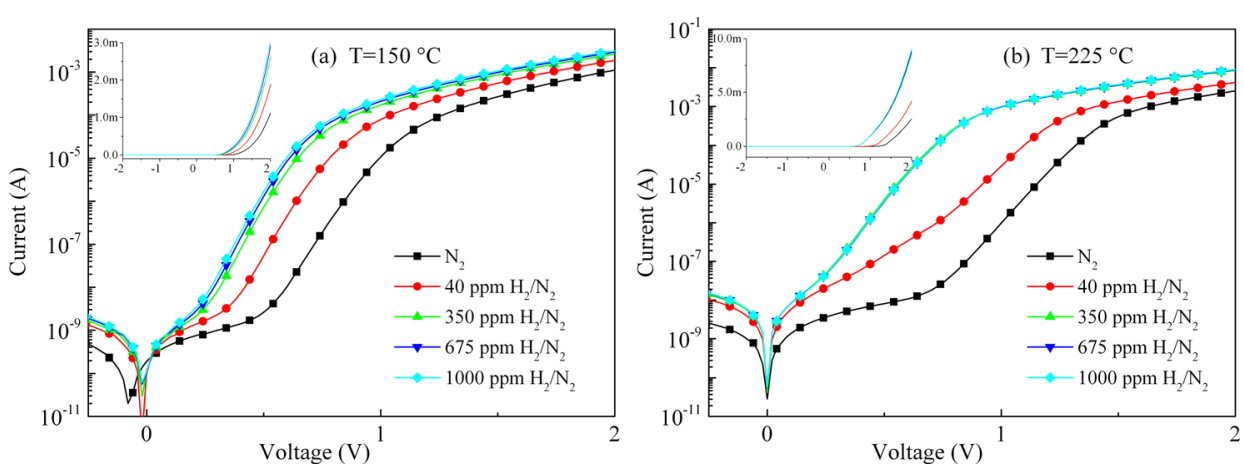

FIG. 1. Current-voltage $(I-V)$ characteristics of the $\mathrm{Pd} / \mathrm{WO} 3 / \mathrm{SiC}$ diode when exposed to $\mathrm{N}_{2}$ and different concentrations of $\mathrm{H}_{2} / \mathrm{N}_{2}$ at (a) $150{ }^{\circ} \mathrm{C}$ and (b) $225^{\circ} \mathrm{C}$ in semi-logarithmic scale. Insets are the linear plots. 
TABLE I. Barrier height and ideality factor of $\mathrm{Pd} / \mathrm{WO}_{3} / \mathrm{SiC}$ diode extracted from the $I-V$ data in the thermal-emission exponential region and parallel resistance-controlled linear region at $150^{\circ} \mathrm{C}$ and $225^{\circ} \mathrm{C}$.

\begin{tabular}{|c|c|c|c|c|c|c|c|c|}
\hline \multirow{3}{*}{$\begin{array}{l}\mathrm{H}_{2} / \mathrm{N}_{2} \\
\mathrm{ppm}\end{array}$} & \multicolumn{4}{|c|}{$150^{\circ} \mathrm{C}$} & \multicolumn{4}{|c|}{$225^{\circ} \mathrm{C}$} \\
\hline & \multicolumn{2}{|c|}{ Exponential } & \multicolumn{2}{|c|}{ Linear } & \multicolumn{2}{|c|}{ Exponential } & \multicolumn{2}{|c|}{ Linear } \\
\hline & $\phi_{\mathrm{B} 0}$ & $n$ & $\phi_{\mathrm{B} 0}$ & $n$ & $\phi_{\mathrm{B} 0}$ & $n$ & $\phi_{\mathrm{B} 0}$ & $n$ \\
\hline 0 & 1.47 & 1.51 & 1.22 & 5.35 & 1.64 & 1.89 & 1.35 & 8.20 \\
\hline 40 & 1.41 & 1.28 & 1.35 & 1.53 & 1.51 & 1.75 & 1.34 & 2.88 \\
\hline 350 & 1.35 & 1.16 & 1.34 & 1.18 & 1.42 & 1.27 & 1.42 & 1.28 \\
\hline 670 & 1.32 & 1.17 & 1.32 & 1.18 & 1.42 & 1.27 & 1.42 & 1.28 \\
\hline 1000 & 1.31 & 1.19 & 1.30 & 1.21 & 1.42 & 1.27 & 1.42 & 1.28 \\
\hline
\end{tabular}

at $150^{\circ} \mathrm{C}$ and even larger than 0.9999 at $225^{\circ} \mathrm{C}$, indicating the highly linear relation between the logarithmic sensitivity and applied voltage.

It should be noted that the linear rising part corresponds to the parallel-resistance-controlled region of the $I-V$ data measured under pure $\mathrm{N}_{2}$, but it corresponds to the thermalemission part under hydrogen gas. The "pseudo" barrier height and ideality factor in the linear region $(0.34 \mathrm{~V}-0.52 \mathrm{~V})$ are also extracted using Eqs. (2) and (3), and shown in Table I. The ideality factor of the device in $\mathrm{N}_{2}$ at $150^{\circ} \mathrm{C}$ and $225^{\circ} \mathrm{C}$ is 5.35 and 8.20 , respectively, far away from the ideal value of one, which can be considered as another proof that the low-current region is controlled by parallel resistance instead of thermal emission. Then, based on the above pseudo values, the simulated slope and intercept according to Eq. (9) are calculated, as denoted by the dash lines in Fig. 2. Excellent agreement between the linear fitting and simulation curve can be seen at both temperatures, especially under $1000-\mathrm{ppm}_{2} / \mathrm{N}_{2}$, thus confirming the validity of Eq. (9).
To predict the maximum value of sensitivity and find the corresponding applied voltage, three steps are used here: First, obtain the slope in the linear rising section with Eq. (9), followed by calculating the related voltage shift $(\Delta V)$, and then, the maximum sensitivity $\left(S_{\max }\right)$ can be instantly estimated, as illustrated in Fig. 3(a).

The data measured in $\mathrm{N}_{2}$ and 1000-ppm $\mathrm{H}_{2} / \mathrm{N}_{2}$ (briefly written as $\mathrm{H}_{2}$ in the following) at $225^{\circ} \mathrm{C}$ is taken as an example, as shown in Fig. 3(b). On the left $y$-axis (logarithmic scale), the bottom two solid lines (curves 1 and 2) are the measured $I-V$ data in $\mathrm{N}_{2}$ and $\mathrm{H}_{2}$ and the top dash line (curve 5) is the corresponding sensitivity. On the right $y$-axis (linear scale), the square and circle dots (curves 3 and 4 ) are the calculated power exponent value for $\mathrm{N}_{2}$ and $\mathrm{H}_{2}$, respectively.

Based on the power exponent and voltage relation in Eq. (4), the $\alpha-V$ curve increases linearly in the low-bias region until reaching a maximum value $\alpha_{\max }$ and then decreases to a constant value due to the effect of series resistance at high bias, as shown by the curve 4 in Fig. 3(b). The peak is marked with $\mathrm{M}_{\alpha}$, and the relevant applied voltage is denoted as $V_{M-\alpha}$.

According to the previous work by $\mathrm{V}$. Mikhelashvili et al., ${ }^{8,13}$ the equations describing the relations between $V_{M-\alpha}, \alpha_{\max }$ and $n, R_{\mathrm{s}}, I_{0}$, can be simplified as follows:

$$
\alpha_{\max } \exp \left(\alpha_{\max }+1\right)=\frac{k T}{q} \frac{n}{I_{s} R_{s}} .
$$

Solving this transcendental equation,

$$
\begin{gathered}
\alpha_{\max }=W\left(\frac{n_{H} k T}{e q I_{s} R_{s}}\right), \\
V_{M-\alpha}=\alpha_{\max } \frac{n_{H} k T}{q},
\end{gathered}
$$

where $W$ is the Lambert function.
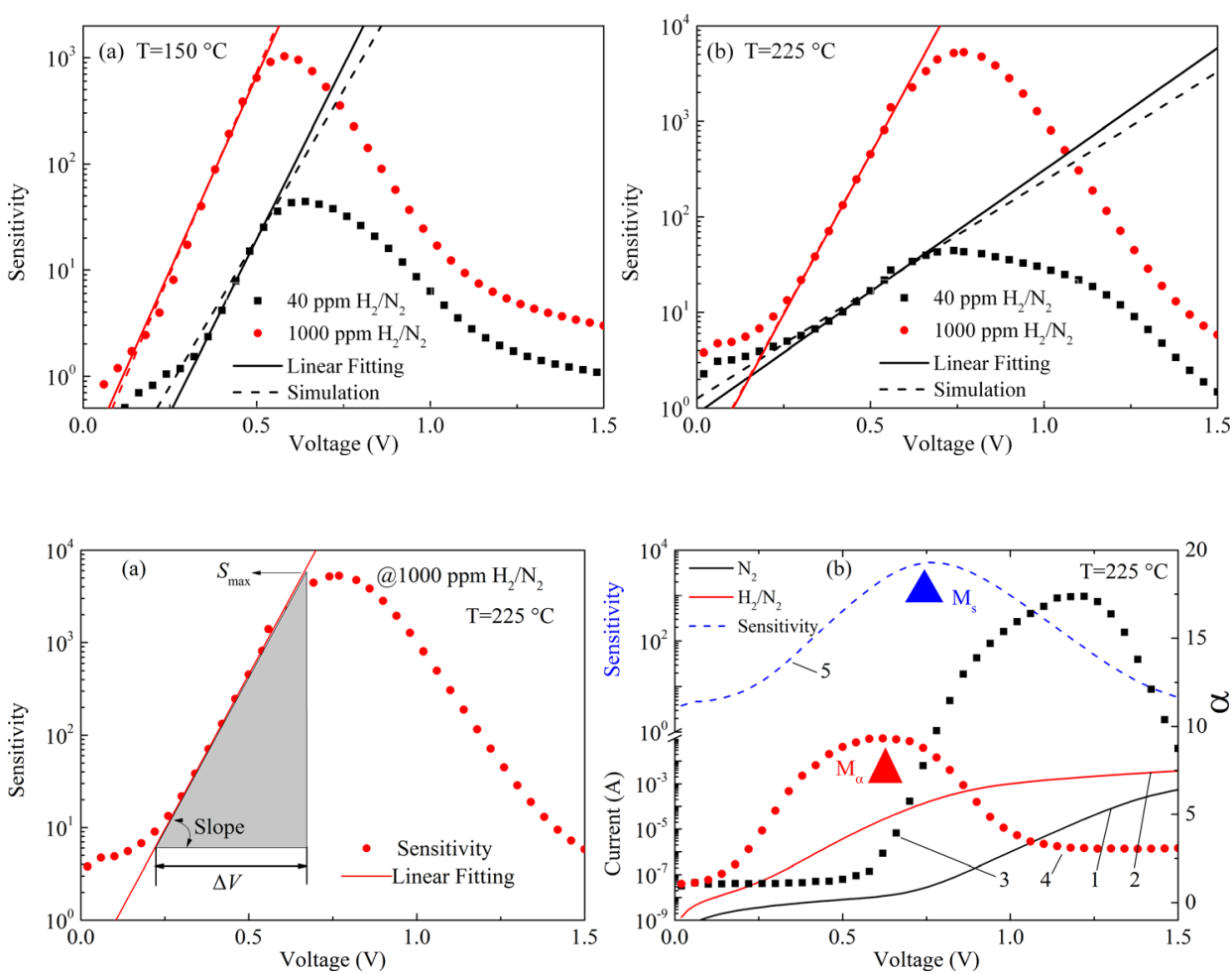

FIG. 2. Sensitivity-voltage $(S-V)$ characteristics of the $\mathrm{Pd} / \mathrm{WO}_{3} / \mathrm{SiC}$ diode when exposed to 40-ppm and 1000ppm $\mathrm{H}_{2} / \mathrm{N}_{2}$ at (a) $150{ }^{\circ} \mathrm{C}$ and (b) $225^{\circ} \mathrm{C}$. The solid line and dash line are linear fitting and simulation data based on the extracted parameters in the linear region.
FIG. 3. (a) Approximate-triangle method for calculating the maximum sensitivity and the applied voltage, (b) Curves 1 and $2-I-V$ data in $\mathrm{N}_{2}$ and 1000 -ppm $\mathrm{H}_{2} / \mathrm{N}_{2}$. Curves 3 and 4 - the corresponding $\alpha-V$ data. Curve 5-the $S$ - $V$ data. Ms and $\mathrm{M}_{\alpha}$ are the points representing the maximum sensitivity and maximum $\alpha$, respectively. 
Because of the parallel resistance in the low-bias region when the diode operates in $\mathrm{N}_{2}$ ambience, the $\alpha-V$ curve stays at the level of $\alpha=1$ before linearly increasing and intersects with the $\alpha-V$ curve under $\mathrm{H}_{2}$, where the maximum sensitivity $S_{\max }$ of the gas sensor is located, marked with $\mathrm{M}_{\mathrm{s}}$, and the corresponding voltage is denoted as $V_{M-S}$. By setting Eq. (6) equal to zero for maximum sensitivity, the intersection point of the two $\alpha$ curves in $\mathrm{H}_{2}$ and $\mathrm{N}_{2}$ (curves 3 and 4) is just located at the same voltage as the maximum sensitivity (curve 5), about $0.75 \mathrm{~V}$ in Fig. 3(b).

Similarly,

$$
V_{M-S}=\alpha_{\max } \frac{n_{N} k T}{q} .
$$

Ignoring the intercept of Eq. (9),

$$
\ln S_{\max }=\frac{q}{k T}\left(\frac{1}{n_{H}}-\frac{1}{n_{N-l}}\right) V_{M-S},
$$

where $n_{N-l}$ is the pseudo-ideality factor in the linear region.

Combining Eqs. (13) and (14), the formula for calculating the maximum sensitivity can be obtained

$$
S_{\max }=\exp \left[\left(1-\frac{n_{H}}{n_{N-l}}\right) \alpha_{\max }\right] .
$$

Then, the above equations can be verified based on the extracted parameters in Table I $\left(n_{H}=1.27, n_{N}=1.89\right.$, $n_{N-l}=8.20, k T / q=0.043$, and $\left.\alpha_{\max }=9.3\right)$, as shown by the curve 4 in Fig. 3(b). The calculated results $\left(V_{M-\alpha}=0.51 \mathrm{~V}\right.$ and $\left.V_{M-S}=0.75 \mathrm{~V}, S_{\max }=2590\right)$ are all in agreement with the experimental data in Fig. 3(b).

In summary, the four important parameters of the sensor $\left(\alpha_{\max }, V_{M-\alpha}, V_{M-S}\right.$, and $\left.S_{\max }\right)$ can be extracted from the conventional parameters of the Schottky diode (ideality factor, saturation current, and series resistance) by simple formulas. They afford guideline to apply a proper operating voltage for Schottky-diode gas sensor and ability to estimate its maximum sensitivity. Moreover, based on the $\alpha-V$ curve, different current-transport mechanisms can be easily identified for different bias voltages.
In this work, a data interpretation technique is presented for analyzing the sensitivity of Schottky-diode gas sensor. This technique makes use of the power exponent parameter $\alpha$ of $I-V$ characteristics and its direct relation with the sensor sensitivity. By studying the voltage dependence of $\alpha$, an exponential relation between the sensitivity and applied voltage in the low-bias regime is demonstrated, and it also allows an easier method to calculate the barrierheight change after the sensor is exposed to a target gas. Experimental confirmation is performed with the most commonly-used MIS structure based on $\mathrm{Pd} / \mathrm{WO}_{3} / \mathrm{SiC}$ measured at different temperatures and hydrogen concentrations. In addition, formulas for calculating the maximum sensitivity and the corresponding applied voltage are derived and verified. These two parameters provide significant assistance for prediction and evaluation on the performance of practical devices.

This work was supported by the CRCG Small Project Funding (No. 201109176240), and the University Development Fund (Nanotechnology Research Institute, No. 00600009) of the University of Hong Kong.

${ }^{1}$ M. S. Shivaraman, I. Lundstrom, C. Svensson, and H. Hammarsten, Electron. Lett. 12, 483 (1976).

${ }^{2}$ T. Hübert, L. Boon-Brett, G. Black, and U. Banach, Sens. Actuators, B 157,329 (2011).

${ }^{3}$ I. Lundström, J. Vac. Sci. Technol., A 14, 1539 (1996).

${ }^{4}$ X. Liu, S. Cheng, H. Liu, S. Hu, D. Zhang, and H. Ning, Sensors 12, 9635 (2012).

${ }^{5}$ K. Potje-Kamloth, Chem. Rev. 108, 367 (2008).

${ }^{6}$ P. Chattopadhyay, J. Phys. D: Appl. Phys. 29, 823 (1996).

${ }^{7}$ J. R. H. Werner, Appl. Phys. A 47, 291 (1988).

${ }^{8}$ V. Mikhelashvili, G. Eisenstein, and R. Uzdin, Solid. State. Electron. 45, 143 (2001).

${ }^{9}$ G. D. Bagratishvili, R. B. Dzhanelidze, D. A. Jishiashvili, L. V. Piskanovskii, A. N. Zyuganov, V. N. Mikhelashvili, and P. S. Smertenko, Phys. Status Solidi A 65, 701 (1981).

${ }^{10}$ E. H. Rhoderick and R. H. Williams, Metal-Semiconductor Contacts (Oxford, Clarendon M.A., 1988).

${ }^{11}$ Y. Liu, J. Yu, and P. T. Lai, Int. J. Hydrogen Energy 39, 10313 (2014).

${ }^{12}$ V. Aubry and F. Meyer, J. Appl. Phys. 76, 7973 (1994).

${ }^{13}$ V. Mikhelashvili, G. Eisenstein, V. Garber, S. Fainleib, G. Bahir, D. Ritter, M. Orenstein, and A. Peer, J. Appl. Phys. 85, 6873 (1999). 